\title{
Procedure Code
}

National Cancer Institute

\section{Source}

National Cancer Institute. Procedure Code. NCI Thesaurus. Code C154626.

Procedure codes indicate the discrete medical interventions and diagnostic testing, such as surgical procedures and lab orders, delivered within a healthcare context. 